\title{
Design and Implementation of Foreign Object Detection Method in Urban Rail Train Driving
}

\author{
Zhang Zhuxi \\ National Transportation Preparation Office \\ Beijing China, 100036
}

\author{
Wang Fei \\ School of Computer and Information Technology \\ Beijing Jiaotong University \\ Beijing, China, 100044
}

\begin{abstract}
The economic development has been accompanied by the continuous increase of the urban population, which has led to an increase in the city's total transportation demand and the urban traffic pressure. As a public service, urban rail transport plays a huge and indispensable role in solving people's travel problems. There are many reasons that affect the operation of urban rail trains, and among which tracking foreign bodies is one of the more common types. So, this paper focuses on the following three tasks: foreign object detection, foreign object classification, and early warning mechanism. Firstly, it designs and implements the detection method of foreign objects in the driving of urban rail vehicles. Secondly, it provides a foreign object detection algorithm to analyze and track the status of foreign objects efficiently by analyzing the track live video generated in the train operation system. Finally, an appropriate alarm processing is proposed according to the situation.
\end{abstract}

Keywords-urban rail transit, detection methods of foreign bodie, support vector machine

\section{INTRODUCTION}

Since the first subway line was formally put into operation, the development of urban rail transit in China has been rapid. The urban rail transit has greatly facilitated people's travel. At the same time, it has also aroused concern for the safety of train operations. There are many factors that affect the safe operation of urban rail trains. Orbital foreign bodies are a very common type. In recent years, the news that foreign bodies affect the operation of orbital trains has been reported from time to time [1]

The influence of track foreign objects on the train cannot be ignored. Rapid detection of foreign bodies on the track and timely removal operations will help guarantee the safety of the train and ensure normal travel. Because of the high speed of rail trains, it is unfeasible to detect foreign objects with the naked eye of the crew. The foreign objects are detected by watching the video surveillance. Due to the subjectivity and psychological status of the observers, the monitoring conclusions are subjective. The effect is also not reliable. The more reliable method is to use related technologies to achieve automatic detection and alarm of foreign objects on the track.

At present, the track foreign object detection technology is mainly applied to the railway field. In a comprehensive view, the current track foreign object detection technology can be divided into two types according to its detection form.

The first is contact detection technology. The core device of the contact foreign matter detection technology is a protective net. The second is non-contact detection technology. In the non-contact detection technology, intelligent video content analysis, infrared detection, radar detection, etc. are three common technologies. Compared with the two detection technologies, the contact detection technology has a low degree of intelligence, and it usually requires human participation in the confirmation of the alarm, which cannot achieve the purpose of automatically identifying the automatic warning, and the related equipment investment is relatively large. Relatively speaking, the non-contact detection technology has a high degree of automation, and the installation of the equipment is flexible and convenient [2]. Of course, this method also has a problem that is greatly affected by environmental factors. In general, both detection methods have their possible and inadequacies. At present, the non-contact detection method is the main research and development direction. One of the current research hotspots in intelligent video content analysis.

The goal of this paper is to design and implement the detection method of foreign objects in the driving of urban rail vehicles. The main content is to use the foreign object detection algorithm to analyze and track the status of foreign objects in a timely manner by analyzing the track live video generated in the train operation system. Appropriate alarm processing according to the situation. This article focuses on the following three tasks: foreign object detection, foreign object classification, and early warning mechanism.

\section{FOREIGN BODY DETECTION}

\section{A. The basic steps of foreign body detection}

Foreign object detection is an application aspect of target detection. Target detection has a long history. The traditional target detection method mainly depends on the subjective judgment of the person. This method is subjective, and due to the problem of work intensity, it often causes the monitoring staff to make wrong judgments, resulting in the consequences. The accuracy is low, and even sometimes it gives wrong results.

With the rapid development of computer science and technology, some image processing related algorithms have been proposed one after another, which greatly improves the efficiency of target detection. In general, target detection mainly depends on the following parameters: color, edge, and corner [3].

In general, the detection of the target detection algorithm 
is often determined by the specific detection scenario and target, but no matter which target is detected, the steps of the algorithm are similar. The basic process of target detection is shown in Figure 1.

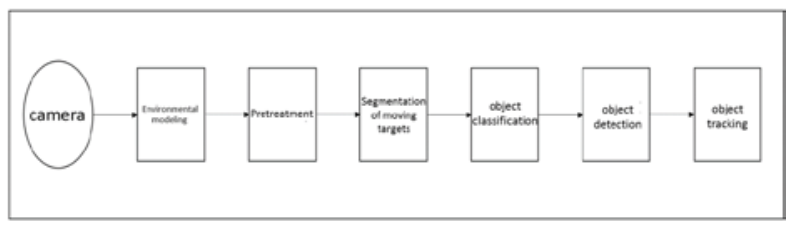

Fig. 1. Target detection basic flow chart

\section{B. Double background modeling}

Foreign object detection refers to the determination of the presence and location of foreign objects from the images captured by surveillance cameras. Under normal circumstances, to judge whether there is foreign matter in a given image, the core idea is to find an object for reference, that is, the background, separate the part that does not belong to the regular background, and extract the target area. Its position is the process of detecting foreign objects. This step is the basis for subsequent visual processing such as image recognition.

At present, the optical flow method, the frame difference method, and the background subtraction method are frequently used methods. Each target detection algorithm has its own advantages and disadvantages. It is difficult to obtain better detection results by adopting one of the detection methods alone. The dual-background modeling method uses multi-frame averaging method and continuous frame difference method to perform background modeling. This method weakens the disadvantages of the frame difference method and further improves the efficiency of foreign object detection.

Dual-background modeling for foreign object detection structure block diagram is shown in Figure 2.

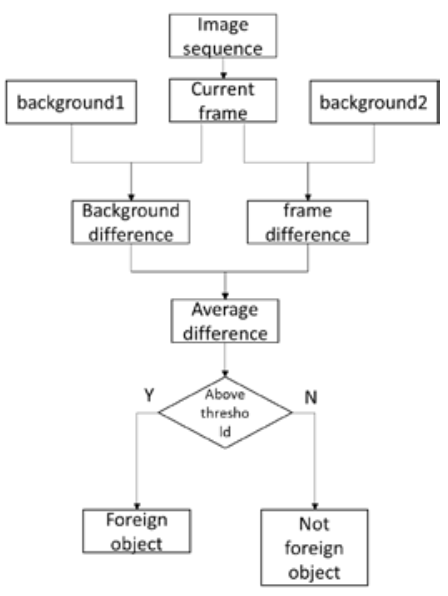

Fig. 2. Dual Background Modeling Background Update Flowchart

\section{Morphological image processing}

In the foreign matter detection process, due to environmental factors and the reasons for the image acquisition device itself, the processed image tends to have some isolated glitches and noises, which greatly affects the determination of foreign objects' contours, positions, and sizes in subsequent steps. The use of mathematical morphology to process images can reduce glitches and noise, and help detect and identify foreign objects [4].

The foreign object image obtained by the double background modeling often cannot directly determine the position of the foreign object and requires further morphological processing operations.

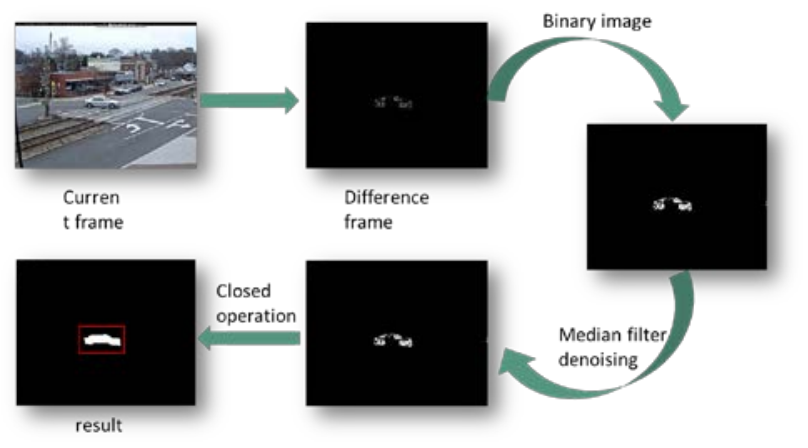

Fig. 3. Further operations

\section{FOREIGN OBJECT RECOGNITION}

Foreign object classification refers to the classification of foreign objects through images to determine the approximate type of foreign objects. The main purpose of this step is to provide preconditions and bedding for subsequent warning work. Foreign object classification mainly involves the following processes: dataset construction, feature selection, and classifier training.

\section{A. Dataset construction}

At the time of training, the data sets used in this paper were manually constructed. There are four main types of foreign objects that need special attention in this article: pedestrians, vehicles, roadblocks, and falling rocks. In the data collection process, 600 images for each type of image, a total of 2400 images are used as the original data of the experiment. Then the HOG features of these pictures are extracted and used together as a training set and a test set of the SVM [5].

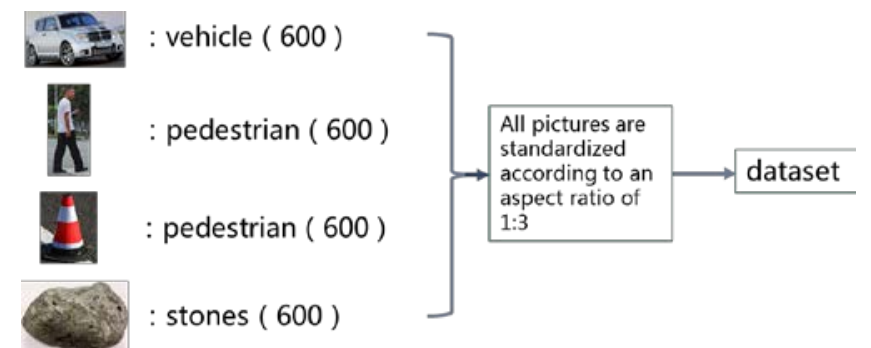

Fig. 4 . Dataset construction

\section{B. Feature selection}

The HOG feature, also called the direction gradient histogram, is often used to detect objects. Compared to other feature description methods, the HOG feature has many characteristics. For example, when the image geometry changes, the HOG feature result can still remain unchanged. This feature also applies to the optical deformation, that is, it can be allowed to be observed. Subjects undergo slight changes without affecting the results. 


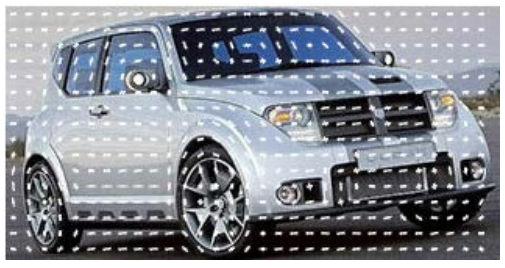

Fig. 5. Hog feature visualization

\section{SVM Classifier}

In the field of machine learning, SVM is a supervised learning model. The so-called supervised learning model refers to the machine learning task that infers functions from the tagged training data set. In terms of popularization, it is necessary to train such models. Use the marked data set. Common supervised learning includes classification and regression. In the problems of pattern recognition, classification and regression analysis, SVM is often the primary choice for solving problems.

In general, SVM has some irreplaceable advantages over other classification algorithms. First, SVM is effective in high-dimensional space. Second, if the number of training samples is small and the feature dimension of the sample is larger than the sample size, SVM is also effective in this case. Therefore, this paper chooses SVM classifier as the classification model.

\section{Classifier training}

In the classifier training process, this article uses a 10fold cross-validation method, which divides the 2,400 images in the data set into ten parts equally. In each training, ten of them are used as a training set for classifier training. The remaining nine data are used as test sets. Each data set takes turns as a test set. The rest is a training set. After each training test, a classifier is obtained according to the correct number of classifiers for the test set. Accuracy, a total of ten training tests, so will get ten different value of accuracy, the accuracy of the final classifier to take the average of ten value of accuracy.

\section{E. Training result}

The results of ten tests are shown in Table I. categories, namely moving foreign bodies and relatively stationary foreign bodies. In the four kinds of foreign bodies, generally speaking, the former two belong to the foreign body of the movement, and the latter two belong to relatively stationary foreign bodies. For foreign objects in motion, if a pedestrian is stumbled in, in a short time, it is possible for the pedestrian to leave the monitoring area by himself. Therefore, there is no need for an immediate alarm operation for such foreign objects, and it should be confirmed again after a certain time interval. The existence of the foreign body, and give early warning according to the existence. For a relatively stationary foreign object, it will stay on once it enters the monitoring area. Therefore, for this type of foreign object, the solution given in this paper is that once the presence of such a foreign object is identified, the system will immediately trigger an alarm.

The early warning mechanism of this paper is shown in Table II.

TABLE II. FOREIGN MATERIAL CLASSIFICATION TABLE

\begin{tabular}{|c|c|c|}
\hline Foreign body state & Type of foreign body & Treatment plan \\
\hline Relatively mobile & Pedestrians, vehicles & $\begin{array}{c}\text { After a certain time } \\
\text { interval, if the } \\
\text { foreign matter is still } \\
\text { there, an alarm is } \\
\text { triggered }\end{array}$ \\
\hline Relatively static & $\begin{array}{c}\text { Roadblocks, falling } \\
\text { stones }\end{array}$ & $\begin{array}{c}\text { An alarm is triggered } \\
\text { upon the detection of } \\
\text { the presence of such } \\
\text { a foreign object }\end{array}$ \\
\hline
\end{tabular}

\section{SYSTEM IMPLEMENTATION}

\section{A. Overall architecture}

In order to realize the track foreign object detection in the computer system, in the experiment, all the code writing work of this paper is carried out in matlab. MATLAB is a commercial mathematics software that is often used for mathematical modeling and analysis of algorithms.

In this experiment, there are a total of two main programs. One is a foreign object detection program for real-time detection of track foreign objects in the video, and the other is a program related to SVM classifier training. The flow of the entire system's program is shown in Figure 6.

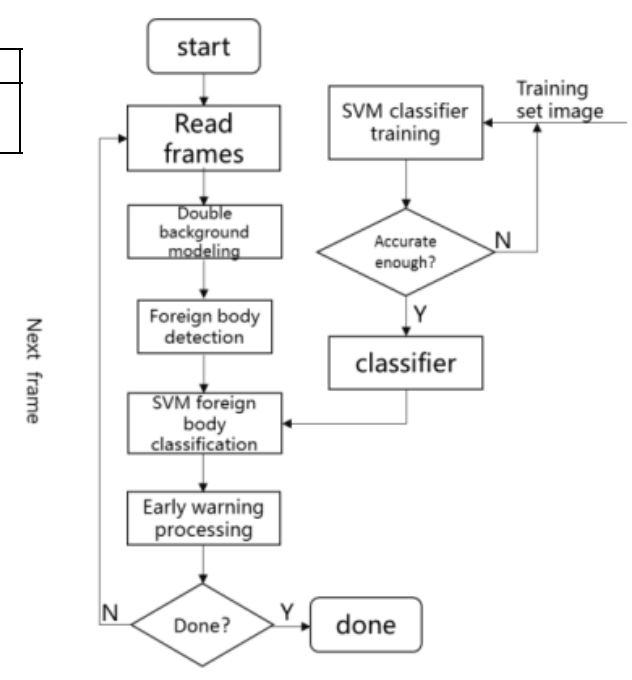

Fig. 6. System flow chart (low chart

TABLE I TRAINING RESULT

\begin{tabular}{|c|c|c|c|c|c|c|c|c|}
\hline Test & 1 & 2 & 3 & 4 & 5 & 6 & 7 & 8 \\
\hline $\begin{array}{c}\text { Accuracy } \\
(\%)\end{array}$ & 99.17 & 100 & 98.75 & 99.58 & 97.50 & 98.75 & 99.58 & 98.75 \\
\hline
\end{tabular}

According to Table I, the average accuracy rate of the classifier training can be $98.71 \%$.

\section{EARLY WARNING MECHANISM}

The alarm is mainly aimed at the possibility that the detected foreign object will potentially affect the orbital operation. Under the condition that certain pre-warning conditions are met, the necessary early warning processing will be carried out to inform the relevant personnel to carry out the necessary foreign object cleaning operations [6].

This paper only studied four common foreign objects on the track, namely pedestrians, vehicles, roadblocks, and rocks. According to the different motion states of foreign bodies, four types of foreign bodies can be divided into two 


\section{B. Running result}

(1) Run the startup file, the program pauses on the first frame of the video, and the mouse manually selects the monitoring area.

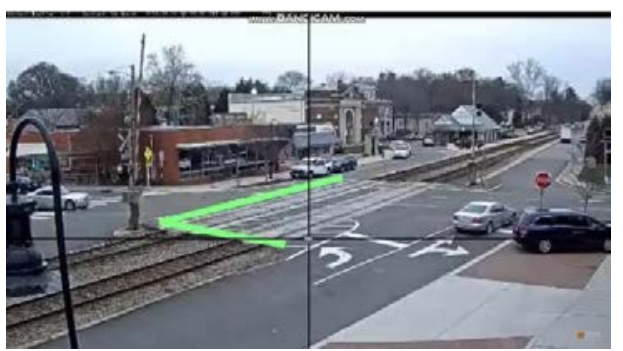

Fig. 7. State 1

(2) After confirming the monitoring area, the foreign object detection system is officially put into operation. The green quadrilateral area is determined by the mouse.

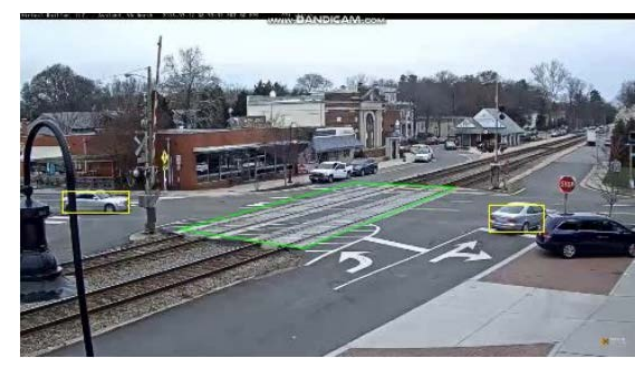

Fig. 8. State 2

(3) When there are moving foreign objects, such as the vehicle entering the monitoring area, the system will use a red box to mark the location of foreign objects in the video.

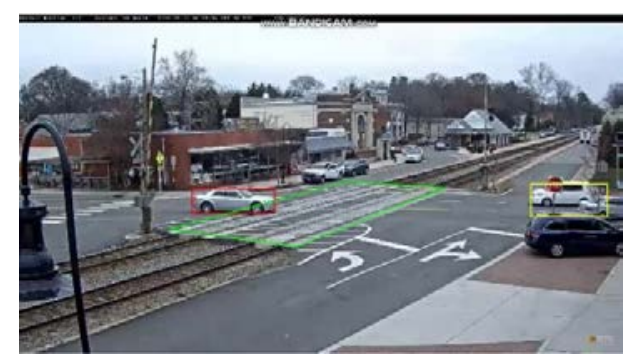

Fig. 9. State 3

(4) The console outputs the warning result based on the detection of foreign objects.

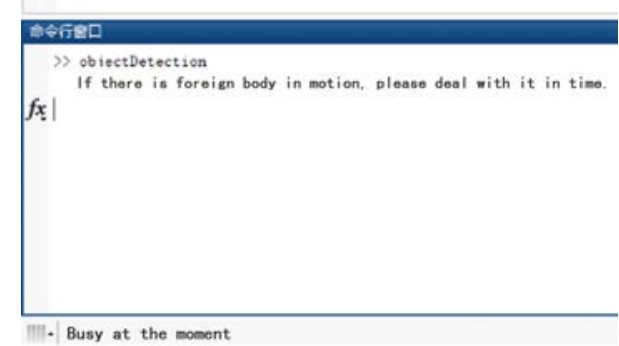

Fig. 10. State 4

\section{DiRECTIONS For IMPROVEMENT AND SUGGESTIONS}

At present, based on the work done in previous chapters, the basic system has been designed and the system has basically fulfilled the expected functions and achieved the expected results. However, as an application program for the actual scene, there are still some problems and shortcomings in adapting to the actual detection environment.

\section{A. Foreign matter detection}

In this paper, a double background modeling method is used to detect and mark the foreign objects in the actual scene. The result of marking is rough. The main purpose is to roughly determine the position of foreign objects. There is no in-depth analysis of the precise shape and movement of foreign objects. Without the corresponding foreign object tracking mechanism, the system built in this paper needs to be modified in the tendency of both foreign object detection and foreign object tracking.

\section{B. Accuracy of foreign material classification}

At present, the system mainly targets four types of foreign objects, and there are few types of foreign objects. In addition, the training set used for training is not large enough. The examples used in this paper are not enough. In the actual complex application scenario, the accuracy of the trained classifier may decrease. For these two problems, we can solve from two aspects. One is the training of classification models, the use of new classification features, such as texture features, color features, design of new kernel functions, adjustment of model parameters, etc., and the other is replacement. A more efficient classification model radically improves classification efficiency and accuracy.

\section{The real-time aspect of the test.}

The operating environment of the system implemented in this article is a windows 8.1 system with 12GB of installed memory and a Core I5 processor. In the running process of the program, the frame rate of the processed video is smaller than the video frame rate before processing. Foreign object detection and foreign object recognition of the video frame cause a large time difference between the video frame and the video frame, resulting in the eventual. The video frame rate seen is decreasing. There are two main reasons for this situation. One reason is that the programming of the program needs to be optimized. Selecting an algorithm with a smaller time complexity to handle it can alleviate this situation to some extent. Another reason is that the performance of the platform on which the program is running is not good enough, and the processing speed of the processor is not fast enough. Selecting a dedicated server loading program can fundamentally greatly increase the speed of the program and improve the real-time performance of the entire detection system.

The above-mentioned problems mainly stem from the simplification of the model and the complexity of the actual scene. At present, the development and application of related technologies such as machine learning and neural networks are becoming more and more widespread. Computer vision algorithms that use these technologies can handle more complex scenes than ever before. Therefore, in the subsequent improvement process of the proposed system, you can consider applying these technologies to them to improve the overall system performance. 


\section{CONCLUSION}

In the actual operation of the train, the track conditions are very complex, and the types of foreign objects appearing are more complex and varied than those envisaged in the research process. The current track foreign object detection technology is diverse. This paper uses the most classical inter-frame difference method. Based on this, a double background modeling method is used to overcome some of the shortcomings of the inter-frame difference method. Using the double background modeling method, the position of the foreign matter is basically determined. Then, the SVM classifier is used to classify the detected foreign objects. At present, this article only studies several types of common foreign objects: pedestrians, vehicles, roadblocks, and rocks. For other types of foreign materials, appropriate extensions can be made during the subsequent research. In order to ensure the reliability of the classification accuracy of the classifier, a 10-fold cross validation method is used in the training of the classifier. After the identification of the foreign matter, the system can perform certain early warning processing according to the foreign matter.

In the implementation of the system in this article, it took a relatively long time in several important aspects. One is the selection of foreign matter detection algorithm, and the other is the training of the classifier. These two parts have a decisive influence on the realization of the final system. In the actual code implementation process, the code implementation logic, code preparation, etc. It will also have a significant impact on the performance of the entire system. In the later in-depth study process, these parts are also the focus of the amendment to discuss the object.
At present, only the detection of foreign objects on the track under the fixed camera conditions has been studied. In the actual scene, the camera operates in various ways. There are fixed-point installations, the camera can be freely typed from multiple angles, and is fixed on the top of the train. Types of. For these different installations of the camera, the methods used in this article also need to be modified. However, in general, it is still possible to imitate the ideas mentioned in the article for foreign object detection, ie, a series of processes such as background modeling, foreign object detection, and foreign object recognition.

\section{REFERENCES}

[1] Dalal N, Triggs B. Histograms of Oriented Gradients for Human Detection[C]// IEEE Computer Society Conference on Computer Vision \& Pattern Recognition. IEEE Computer Society, 2005:886893.

[2] Ojala T, Pietikainen M, Harwood D. Performance evaluation of texture measures with classification based on Kullback discrimination of distributions[C]// Iapr International Conference on Pattern Recognition, 1994. Vol. 1 - Conference A: Computer Vision \& Image Processing. IEEE, 2002:582-585 vol.1.

[3] PapageorgiouCP, Oren M Poggio T. A general framework for object detection[C]//ComputerVision , 1998.Sixth International Conference on.IEEE, $1998: 555-562$

[4] Cortes, C. \& Vapnik, V. Mach Learn (1995) 20: 273. https://doi.org/10.1007/BF00994018

[5] Zhao Q, Li F, Liu X. Real-Time Visual Odometry Based on Optical Flow and Depth Learning[C]// International Conference on Measuring Technology and Mechatronics Automation. IEEE Computer Society, 2018:239-242.

[6] Liu M, Liang N. Detection of Moving Target Using Improved Optical Flow Method[C]// Software Engineering. IEEE, 2013:311315. 